\section{Nachbesetzung eines Vertragsarztsitzes in einer Berufsausübungsgemeinschaft - Frage des Bestehens einer fortführungsfähigen Praxis}

\section{Einleitung}

Wenn ein Vertragsarzt seine Tätigkeit beenden und seinen Versorgungsauftrag auf einen Nachfolger übertragen möchte, steht ihm in Planungsbereichen, die für die betreffende Arztgruppe wegen Überversorgung gesperrt sind, das sog. Nachbesetzungsverfahren nach $\S 103$ Abs. 3a S. 1, Abs. 4 S. 1 SGB V zur Verfügung.

Nach der gesetzlichen Konzeption ist in überversorgten Planungsbereichen zwar die Nachbesetzung von Vertragsarztsitzen im Grundsatz unerwünscht, jedoch lässt der Gesetzgeber es mit den Regelungen des Nachbesetzungsverfahrens gleichwohl zu, dass ein bestehender - für die Versorgung nicht erforderlicher - Vertragsarztsitz nachbesetzt werden kann. Damit berücksichtigt er die finanziellen Interessen des bisherigen Praxisinhabers bzw. seiner Erben. Weil typischerweise die Arztpraxis nicht veräußert werden kann, wenn der Erwerber den mit ihr verbundenen Sitz nicht erhält, bedarf es der Zulassung des Erwerbers. Nicht der Vertragsarztsitz selbst, sondern nur die Arztpraxis mit ihren materiellen und immateriellen Werten ist dagegen veräußerbar. ${ }^{1}$

Das Nachbesetzungsverfahren kann von Vertragsärzten, die in einer Einzelpraxis oder auch in einer Berufsausübungsgemeinschaft (BAG) tätig sind, in Anspruch genommen werden. Vertragsärzte, die in einer Einzelpraxis tätig sind, können auf ihren Nachfolger und den zu ermittelnden Praxiswert nur geringen Einfluss nehmen, sondern sind weitgehend an die Auswahlund Bewertungsentscheidungen der Zulassungsgremien gebunden. Das Bundessozialgericht (BSG) hat demgegenüber in mehreren Entscheidungen betont, dass den in einer BAG tätigen Ärzten ein besonderer Schutz im Rahmen der Ausschreibung und Nachbesetzung eines aus der BAG ausscheidenden Vertragsarztes zusteht.
Im Rahmen einer aktuellen Entscheidung vom 27.06.2018 (Az.: B 6 KA 46/17 R) hat das BSG der BAG eine besondere Stellung im Rahmen der von den Zulassungsgremien zu beurteilenden Frage eingeräumt, ob die Durchführung eines Nachbesetzungsverfahrens für einen Versorgungsauftrag auch dann verlangt werden kann, wenn dieser wegen unzureichender Teilnahme an der vertragsärztlichen Versorgung im Grunde nicht fortführungsfähig ist.

\section{Sachverhalt der BSG- Entscheidung}

In dem vom BSG entschiedenen Verfahren hatte eine Ärztin auf Nachbesetzung eines Versorgungsauftrags eines verstorbenen Arztes geklagt. Die Ärztin war Mitglied einer BAG, die seit dem Tod eines BAG-Partners noch aus 2 mit vollem Versorgungsauftrag tätigen Chirurginnen bestand. Der verstorbene Arzt war in den Quartalen vor Meldung seiner Erkrankung lediglich im Umfang von ca. $10 \%$ seines vollen Versorgungsauftrags tatsächlich tätig gewesen. Die Gesamtfallzahlen der BAG entsprachen im entscheidungsrelevanten Zeitraum hingegen etwa der durchschnittlichen Fallzahl einer BAG mit 3 im vollen Umfang tätigen Ärzten. Der Zulassungsausschuss hatte die Durchführung eines Nachbesetzungsverfahrens für einen vollen Versorgungsauftrag wegen unzureichender Teilnahme des verstorbenen Arztes an der vertragsärztlichen Versorgung abgelehnt und lediglich der Nachbesetzung hinsichtlich eines halben Versorgungsauftrags zugestimmt. Die Revision der Ärztin hatte Erfolg, da die Ablehnung der Durchführung des Nachbesetzungsverfahrens durch den ZA nach Auffassung des BSG rechtswidrig war. Der Zulassungsausschuss wurde verpflichtet unter Beachtung der Rechtsauffassung des BSG - erneut über den Antrag auf Durchführung eines Nachbesetzungsverfahrens zu entscheiden.

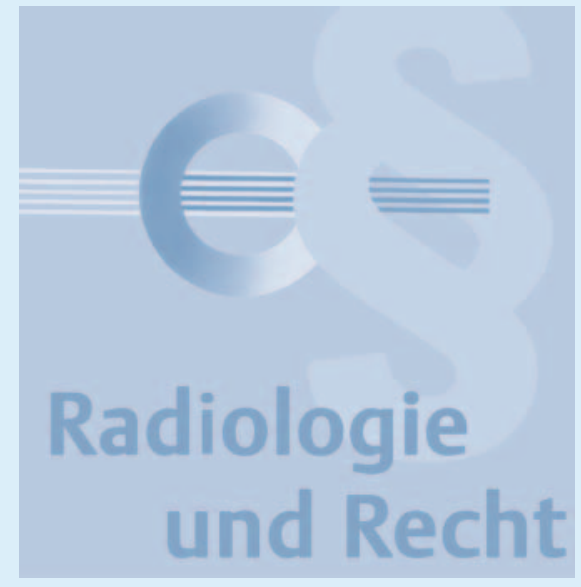

\section{Stellung der BAG im Nachbesetzungsverfahren}

Die BAG (bis zum Inkrafttreten des Vertragsarztrechtsänderungsgesetzes - VÄndG vom 01.07.2007: Gemeinschaftspraxis) zeichnet sich dadurch aus, dass in ihr mehrere Ärzte gleicher oder ähnlicher Fachrichtungen in gemeinsamen Räumen mit gemeinsamem Personal und gemeinsamer Datenverarbeitung unter gemeinsamer Abrechnung die ärztliche Tätigkeit ausüben. Die BAG stellt dabei eine eigene Rechtspersönlichkeit dar. In ihrem äußeren Tätigwerden wird die BAG Vertragspartner der durch ihre Gesellschafter oder deren Angestellte mit den Patienten geschlossenen Behandlungsverträge und dadurch als juristische Person berechtigt und verpflichtet. Ein Zusammenschluss ist jedoch nur in der Form der Gesellschaft bürgerlichen Rechts (GbR) gemäß §§ 705 ff. BGB oder der Partnerschaftsgesellschaft nach dem PartGG zulässig. Demgegenüber hat das BSG mit Urteil vom 15.08.2012² klargestellt, dass es Vertragsärzten in Einzeloder Gemeinschaftspraxen nicht erlaubt ist, ihre Tätigkeit in der Rechtsform einer juristischen Person wie der $\mathrm{GmbH}$ auszuüben, da das SGB V und die Ärzte-ZV hierfür keine Regelungen vorsehen und die Regelung für MVZ in $§ 95$ Abs. 1a S. 1 SGB V auf Vertragsärzte nicht entsprechend angewandt werden kann. Die BAG hat, anders als das Medizinische Versorgungszentrum (MVZ), keinen eigenen Zulassungsstatus; ihr statusrelevanter Bescheid ist die Genehmigung nach $\S 33$ Abs. 2 Zulassungsverordnung für Vertragsärzte (Ärzte-ZV). Maßgeblich für die Entfal-

2 BSG, Urt. v. 15.08.2012, Az.: B 6 KA 47/11 R, NZS 2013, 155. 
tung vertragsärztlicher Tätigkeit in der BAG ist mithin die vertragsärztliche Zulassung ihrer Mitglieder. Die Rechtsprechung des BSG hat indessen den Status der BAG in verschiedener Hinsicht dem Zulassungsstatus des Arztes angenähert. Dies kommt besonders deutlich in den Entscheidungen zur Nachbesetzung eines Arztsitzes zum Ausdruck. ${ }^{3}$

Wie das BSG feststellt, genießt auch die vertragsärztliche Tätigkeit in einer BAG den Schutz des Grundrechts der Berufsausübungsfreiheit aus Art. 12 Abs. 1 GG. Daher ist das Interesse der verbliebenen BAG-Mitglieder an der Nachbesetzung eines frei gewordenen Vertragsarztsitzes in aller Regel schutzwürdig. Nur durch eine solche Berücksichtigung ist nach Ansicht des BSG eine hinreichende Gleichbehandlung mit MVZ zu gewährleisten, denen eine Nachbesetzung von Arztstellen ohne Prüfung von Versorgungsgesichtspunkten nach $\S 103$ Abs. 4a S. 3 SGB V möglich ist. ${ }^{4}$

Insbesondere aber aufgrund der Regelung in $\S 103$ Abs. 6 S. 2 SGB V , wonach bei einer Nachbesetzung eines Vertragsarztsitzes innerhalb einer BAG die Interessen des oder der in der Praxis verbleibenden Vertragsärzte „bei der Bewerberauswahl angemessen zu berücksichtigen“ sind, hat das BSG dem rechtlichen und wirtschaftlichen Bestand der BAG im Nachbesetzungsverfahren einen hohen Stellenwert eingeräumt.

Bereits mit Urteil vom 25.11.1998 (Az.: B 6 KA 70/97R) hatte das BSG entschieden, dass aufgrund der in $\S 103$ Abs. 6 Satz 1 SGB $\vee$ geregelten entsprechenden Anwendung des Abs. 4 auf die BAG den in der Praxis verbleibenden Vertragsärzten die Befugnis zusteht, die Ausschreibung zu beantragen, wie dies in Abs. 4 Satz 1 für den ausscheidenden Vertragsarzt bzw. die zur Verfügung über die Praxis berechtigten Erben vorgesehen ist. In einer weiteren Entscheidung vom 29.09.1999 (Az.: B 6 KA 1/ 99 R) hat das BSG die Privilegierung der verbleibenden Ärzte einer Gemeinschaftspraxis sodann auch auf das in $\S 103$ Abs. 4 Satz 4 SGB V geregelte Auswahlverfahren ausgedehnt und entschieden, dass die Inte-

3 vgl. BSGE 115, $57=$ SozR $4-2500 \S 103$

Nr. 13.

4 BSG, Urt. v. 27.06.2018, Az.: B 6 KA 46/17 R, Rn. 37. ressen der in einer BAG verbleibenden Vertragsärzte auf Fortführung einer BAG in einer bestimmten gewachsenen und im Hinblick auf die apparativtechnische und personelle Ausstattung der Praxis sowie unter Berücksichtigung der Zahl der zu versorgenden Patienten angemessenen Größe nicht gewahrt werden können, wenn im Wege des Nachbesetzungsverfahrens ein Arzt zugelassen wird, der sich an der gemeinsamen Ausübung der vertragsärztlichen Tätigkeit explizit nicht beteiligen will. Des Weiteren hat das BSG in einer Entscheidung vom 05.11.2003 (Az.: B 6 KA 11/03 R) klargestellt, dass in Fällen, in denen sich mehrere annähernd gleich geeignete Ärzte um die Nachfolgezulassung in einer BAG bewerben, die Zulassungsgremien den Arzt bevorzugen dürfen, mit dem der in der BAG verbleibende Arzt die BAG fortführen will und der die Gewähr für eine längerfristige Kooperation bietet.

Mit der Entscheidung vom 27.06.2018 stellt das BSG nun fest, dass die Zulassungsgremien im Rahmen der Nachbesetzung grundsätzlich eine Nachbesetzung mit einem vollen Versorgungsauftrag ermöglichen müssen, wenn die Zulassung eines Arztes mit einem vollen Versorgungsauftrag geendet hat oder beendet werden soll. Die vom BSG hierzu aufgestellten Grundsätze haben weitreichende Konsequenzen für die Zulassungsgremien, wie nachfolgend gezeigt werden soll.

\section{Das Kriterium der fortfüh- rungsfähigen Praxis im Nachbesetzungsverfahren}

Zur Durchführung des Nachbesetzungsverfahrens bedarf es eines wirksamen Antrags an den zuständigen Zulassungsausschuss, dieser bedeutet das Ende der Zulassung des bisherigen Zulassungsinhabers. Es muss sich zudem um eine nachbesetzungsfähige Zulassung handeln und die zur Nachbesetzung vorgesehene Praxis muss fortführungsfähig sein.

Hinsichtlich des Kriteriums der Fortführungsfähigkeit einer Praxis gilt im Rahmen der Einzelpraxis, dass zur Fortführung ein gewisses Praxissubstrat vorhanden sein muss. Dieses kann in dem Vorhandensein von Räumlichkeiten, Ausstattung und Personal, sowie der tatsächlichen Entfaltung vertragsärztlicher Tätigkeit unter Zuhilfenahme der Vorgenannten bestehen. Wenn es an all dem fehlt, existiert keine Praxis mehr, die fortgeführt werden könnte. ${ }^{5}$

In der Entscheidung des BSG vom 27.06.2018 hatte das Sozialgericht die Klage zunächst mit der Begründung abgewiesen, dass nach dem Ausscheiden des Chirurgen keine fortführungsfähige Praxis mehr vorhanden gewesen sei. Im Zulassungs- und Nachbesetzungsverfahren sei nicht die BAG als Rechtspersönlichkeit, sondern lediglich deren einzelne Mitglieder maßgeblich. Der Chirurg sei als eigentlicher Träger der vertragsärztlichen Zulassung nur sehr eingeschränkt oder nicht tätig gewesen und daher sei, mangels eigenen Praxissubstrats und mangelnder Teilnahme an der vertragsärztlichen Versorgung, seine Praxis weggefallen. Die Tatsache, dass die BAG insgesamt die durchschnittlichen Fallzahlen dreier in vollem Umfang tätiger Ärzte der Fachgruppe erreichte, sei unmaßgeblich. Im Kern der Entscheidung des BSG lagen daher die Fragen, ob in Form der vorliegend weiterbestehenden BAG eine im Wege des Nachbesetzungsverfahrens fortführungsfähige Praxis bestand und ob der ZA den nachzubesetzenden Versorgungsauftrag im Rahmen des Nachbesetzungsverfahrens wegen Nichterfüllung um die Hälfte kürzen durfte.

Im Rahmen der Prüfung der Fortführungsfähigkeit einer Praxis zum Zeitpunkt der Beantragung der Nachbesetzung ist nach Ansicht des BSG maßgeblich, ob eine Anknüpfung des Nachfolgers an die bisher in der BAG entfaltete vertragsärztliche Tätigkeit noch möglich ist. Dabei muss die BAG als Rechtseinheit betrachtet werden. Für diese Betrachtungsweise sprechen nach Auffassung des BSG verschiedene Eigenschaften einer BAG, die diese von einer Einzelpraxis unterscheiden.

Einerseits wird der Behandlungsvertrag in einer BAG nach § 630a Abs. 1 BGB nicht zwischen konkretem Behandler und Patienten geschlossen. Vielmehr tritt die BAG an die

5 BSGE $115,57=$ SozR $4-2500 \$ 103$ Nr. 13 , RdNr. 35; BSGE 85, 1, 5 = SozR 3 - $2500 \S 103$ Nr. 5 S. 32. 
Stelle des Behandlers. Im Zuge der Behandlung durch eine BAG ist gerade auch der Wechsel des behandelnden Arztes kennzeichnend. Im Abrechnungswesen wird zudem die abrechenbare Leistung nicht durch ein Mitglied abgerechnet, sondern durch die BAG als Ganzes. Weiterhin ist der gesamte Praxisbetrieb mit Einrichtung, Behandlungsinstrumenten, Personal etc. der BAG zugeordnet.

Mithin ist nach Ansicht des BSG eine BAG grundsätzlich solange als fortführungsfähige Praxis anzusehen, wie sie personelle und sachliche Mittel zur Entfaltung vertragsärztlicher Tätigkeit bereithält und so ein Praxisnachfolger des ausscheidenden BAG-Mitglieds unmittelbar seine vertragsärztliche Tätigkeit aufnehmen kann. Besteht zum Zeitpunkt der Beantragung des Nachbesetzungsverfahrens eine aktive BAG, so muss der Zulassungsausschuss im Rahmen der Einleitung eines Nachbesetzungsverfahrens auch an diese als fortführungsfähige Praxis des ausscheidenden Mitglieds anknüpfen. ${ }^{6}$

Das BSG stellt in seiner Begründung insbesondere auf die Bedeutung einer kooperativen Berufsausübung ab. So sei für die BAG typisch, dass ihre Mitglieder vorübergehend oder auch dauerhaft nicht in gleichem zeitlichem Umfang in der gemeinsamen Praxis tätig sind. Die vertragsärztliche Tätigkeit in einer BAG werde häufig gerade gewählt, weil innerhalb der Kooperation flexibel auf wechselnde Lebenssituationen reagiert werden kann. Zu den Vorzügen der kooperativen Ausübung der vertragsärztlichen Tätigkeit gehöre auch, dass selbst länger andauernde krankheitsbedingte Leistungseinschränkungen durch andere Mitglieder der BAG aufgefangen werden können. Darin liege von vornherein kein Missbrauch von Gestaltungsmöglichkeiten.

\section{Halbierung des}

\section{Versorgungsauftrags im Nachbesetzungsverfahren?}

Vorliegend hatte der ZA im Nachbesetzungsverfahren den durch Verzicht des

6 BSG, Urt. v. 27.06.2018, Az.: B 6 KA 46/17 R, Rn. 32. dritten BAG-Mitglieds freigewordenen Vertragsarztsitz nicht im vollen Umfange, sondern lediglich hälftig nachbesetzt. Hierzu vertrat das BSG die Auffassung, dass der Zulassungsausschuss dazu verpflichtet sei, im Rahmen des Nachbesetzungsverfahrens „[...] grundsätzlich eine Nachbesetzung mit einem vollen Versorgungsauftrag [zu] ermöglichen, wenn die Zulassung des Arztes mit einem vollen Versorgungsauftrag geendet hat oder beendet werden soll. "7 Mithin ist die Nichterfüllung des Versorgungsauftrags durch das ausscheidende Mitglied der BAG zumindest im Hinblick auf das Nachbesetzungsverfahren unbeachtlich.

Dies bedeutet nach Ansicht des BSG jedoch nicht, dass der zuständigen Kassenärztlichen Vereinigung, bei Nichterfüllung des Versorgungsauftrags durch ein Mitglied einer BAG, keine Möglichkeiten der Sanktionierung eröffnet wären. Allerdings stehen diese nur im Rahmen des entsprechenden Verfahrens, d. h. dem der Zulassungsentziehung gem. §95 Abs. 6 Satz 1 SGB V und nicht im Rahmen des Nachbesetzungsverfahrens, zur Verfügung. Um einen Missbrauch der Gestaltungsmöglichkeiten innerhalb einer BAG zu verhindern, indem etwa eine BAG reine „Zählmitglieder" aufnimmt, um eine übermäßige Ausdehnung der vertragsärztlichen Tätigkeit zu verdecken, muss daher ein Vertragsarzt kontinuierlich in nennenswertem Umfang an der vertragsärztlichen Versorgung teilnehmen. ${ }^{8}$ Andernfalls kann ihm auch in einer BAG die Zulassung ganz oder teilweise entzogen werden.

\section{Zur Ablehnung der Nachbesetzung aus} Versorgungsgründen

Der ZA hatte vorliegend eine positive Feststellung der fehlenden Erforderlichkeit der Nachbesetzung zwar unterlassen, das BSG widmete sich jedoch gleichwohl der Frage, ob vorliegend gem. §103 Abs. 3a S. 3 SGB V die Nachbesetzung aus Kapazitätsplanungsgründen hätte abgelehnt werden können.

7 BSG, Urt. v. 27.06.2018, Az.: B 6 KA 46/17 R Rn. 32

8 BSG, Urt. v. 27.06.2018, Az.: B 6 KA 46/17 R, Rn. 33.
In wegen Überversorgung gesperrten Planungsbereichen ist grundsätzlich keine Nachbesetzung freiwerdender Vertragsarztsitze erforderlich. Ausnahmsweise kann eine Nachbesetzung gleichwohl erfolgen, wenn dadurch ein besonderer räumlicher, diagnostischer oder therapeutischer Bedarf innerhalb des Planungsbereichs gedeckt werden kann. Insbesondere ist vor der Ablehnung einer Nachbesetzung aus bedarfsplanungsrechtlichen Gründen ein schonender Ausgleich gegenüber den verbleibenden Mitgliedern der BAG herzustellen. Das BSG führt hier insbesondere ein "[...] bestimmtes Versorgungsangebot [der BAG] an, das ohne Nachbesetzung nicht aufrechterhalten werden kann" "9 und so bis hin zur Existenzvernichtung der gesamten BAG führen kann.

Im Hinblick auf die Versorgungsrelevanz eines Versorgungsauftrags stellt das BSG auf die durchschnittlichen Fallzahlen der BAG berechnet aus der Addition der Individualversorgungsaufträge der Mitglieder - ab. Vorliegend konnten trotz des Ausfalls eines Mitglieds die durchschnittlichen Fallzahlen einer BAG mit 3 voll tätigen Ärzten erreicht werden, sodass auf eine Versorgungsrelevanz des nachzubesetzenden vollen Versorgungsauftrags zu schließen sei.

\section{Ergebnis}

Durch das vorliegende Urteil des BSG werden die Rechte der in einer BAG kooperativ zusammenarbeitenden Vertragsärzte weiter gestärkt. Im Gegensatz zu einer Einzelpraxis müssen die KVen und die Zulassungsgremien hinnehmen, dass einzelne Vertragsärzte ihren Versorgungsauftrag aus nachzuvollziehenden Gründen auch längerfristig nicht erfüllen. Dies wird zukünftig auch Auswirkungen auf die unlängst im Rahmen des Terminserviceund Versorgungsgesetzes (TSVG) beschlossene Verschärfung der Prüfung der Einhaltung der Versorgungsaufträge durch die KVen sowie auf die Weiterleitung der Ergebnisse an und mögliche Sanktionierung durch die Zulassungsgremien in $§ 95$ Abs. 3 SGB $\vee$ haben.

9 BSG, Urt. v. 27.06.2018, Az.: B 6 KA 46/17 R, Rn. 37. 
Mit seiner Entscheidung führt das BSG seine Rechtsprechungslinie zur BAG fort und betont die eigenständige Bedeutung des Status der BAG. Im Rahmen von Nachbesetzungsverfahren in einer BAG wird sich die Frage nach einer fortführungsfähigen Praxis zukünftig häufig erübrigen, sofern die anderen Mitglieder den üblichen Praxisbetrieb im Rahmen ihrer Möglichkeiten aufrechterhalten haben. Im Rahmen des Nachbesetzungsverfahrens in einer BAG ist damit der Versorgungsauftrag grundsätzlich auch in voller Höhe nachzubesetzen, auch wenn das Mitglied diesen vor seinem Ausscheiden nicht vollständig erfüllt hat.

Praktisch bedeutet dies, dass denjenigen Vertragsärzten, die aus Alters- oder sonstigen Gründen nach § 103 Abs. 3a SGB V auf ihre Zulassung verzichten, bzw. den Erben die wirtschaftliche Verwertung der de facto Vermögensposition der „vertragsärztlichen Zulassung“ im Rahmen der Praxisnachfolge sozial- und damit auch zivilrechtlich gesi- chert wird. Weiterhin dienen die Feststellungen des BSG auch den verbliebenen BAG-Mitgliedern. Diese haben unter Umständen die Möglichkeit, den freigewordenen Vertragsarztsitz selbst zu erhalten und die vertragsärztliche Tätigkeit durch angestellte Ärzte weiterführen zu lassen, mithin auch den Weiterbestand der Praxis und deren wirtschaftliche Grundlage langfristig, auch zum Wohle der Patientenversorgung, zu sichern.

Auch im Rahmen der Entscheidung über die Frage der Erforderlichkeit der Nachbesetzung eines Vertragsarztsitzes aus Versorgungsgründen haben die Zulassungsausschüsse das schutzwürdige Interesse der verbleibenden BAG-Mitglieder an einer Nachbesetzung zu berücksichtigen. Damit nähert das BSG die Rechtsstellung von Berufsausübungsgemeinschaften weiter an diejenige von Medizinischen Versorgungszentren an, indem es den Bestandsschutz und die Position der BAG als einheitliche
Rechtspersönlichkeit gegenüber der KV verstärkt. Bereits mit der Entscheidung vom 04.05.2016 hatte das BSG festgestellt, dass die Genehmigung für die Anstellung eines Arztes in einer BAG nicht einem der Mitglieder, sondern der BAG selbst zu erteilen ist. ${ }^{10}$

Prof. Dr. Peter Wigge

Rechtsanwalt

Fachanwalt für Medizinrecht

Florian Markus Dawe, B. A.

Wissenschaftlicher Mitarbeiter

Rechtsanwälte Wigge

Scharnhorststraße 40

48151 Münster

Tel.: (0251) $53595-0$

Fax: (0251) 53 595-99

Email: kanzlei@ra-wigge.de

www.ra-wigge.de 